\title{
Neoadjuvant anti-PD-1 immunotherapy for recurrent glioblastoma
}

\author{
Dana Mitchell, Mahua Dey \\ Department of Neurosurgery, Indiana University School of Medicine, IU Simon Cancer Center, Indianapolis, Indiana, USA \\ Correspondence to: Mahua Dey, MD. Indiana University-Purdue University Indianapolis (IUPUI), Neuroscience Building, 320 W 15 th Street \\ NB400A, Indianapolis, IN 46202, USA. Email: mdey@iu.edu. \\ Comment on: Cloughesy TF, Mochizuki AY, Orpilla JR, et al. Neoadjuvant anti-PD-1 immunotherapy promotes a survival benefit with intratumoral \\ and systemic immune responses in recurrent glioblastoma. Nat Med 2019;25:477-86.
}

Submitted May 08, 2019. Accepted for publication May 20, 2019.

doi: $10.21037 /$ tcr.2019.05.24

View this article at: http://dx.doi.org/10.21037/tcr.2019.05.24

In a healthy host, the programmed cell death (PD) pathway serves to regulate $\mathrm{T}$ cell activity in order to prevent damage secondary to chronic inflammation and the development of autoimmunity. This pathway is often exploited by human cancers to suppress infiltrating cytotoxic $\mathrm{T}$ lymphocytes (CTLs) and evade the anti-tumor immune response (1). Cancers, including glioblastoma (GBM), upregulate the expression of PD-L1, which upon binding to PD-1 expressed on the surface of $T$ cells, results in the attenuation of $\mathrm{T}$ cell activity (2). The resulting attenuation of the antitumor $\mathrm{T}$ cell response is achieved through the suppression of $\mathrm{T}$ cell proliferation and cytokine release as well as the induction of $\mathrm{T}$ cell anergy, promotion of $\mathrm{T}$ cell apoptosis and expansion of the regulatory $\mathrm{T}$ cell (Tregs) population $(1,2)$.

While anti-PD-1 therapy has demonstrated clinical benefit in other types of cancer, previous clinical trials investigating PD-1/PD-L1 axis inhibition in the treatment of patients with GBM have demonstrated limited efficacy (1,3-5). CheckMate 143 (NCT02017717) was the first randomized phase III clinical trial to investigate the use of the anti-PD-1 monoclonal antibody, nivolumab, in the treatment of patients with recurrent GBM, but unfortunately, in comparison to bevacizumab (antiVEGF), it failed to prolong overall survival (OS) and that arm of study was subsequently closed (1). Despite failing to demonstrate improved OS, the CheckMate 143 trial brought to light a small subset of patients who responded to treatment with nivolumab and exhibited a significantly longer duration of response compared to patients receiving bevacizumab (1). These findings suggested that the use of PD-1/PD-L1 axis inhibition in the treatment of patients with GBM was potentially still feasible. The question now became, how and for whom?

To investigate the potential of neoadjuvant PD-1 blockade on altering the functional immune landscape and ultimately, its impact on OS, Cloughesy et al. designed a multi-institutional, randomized, open label pilot study of pembrolizumab in patients with surgically resectable recurrent GBM (6). What they found, could be a game-changer in the use of checkpoint inhibitors in the treatment of GBM. In the study authors compared data from patients receiving neoadjuvant $(n=15)$ to those receiving only adjuvant pembrolizumab $(n=15)$. Not only did their results demonstrate that pembrolizumab was having biological effect, but patients who received neoadjuvant pembrolizumab demonstrated a statistically significant improvement in OS (13.2 vs. 6.3 months for adjuvant group). While the study was small, was powered for tissue analysis, not progression-free survival (PFS) or OS, and most of the patients had died by the end of the study, Cloughesy et al. thoroughly accounted for many of the potential confounding variables (e.g., IDH mutation status, MGMT methylation status, steroid administration, etc.), and demonstrated that the use of anti-PD-1 therapy does, in fact, have a potential role in the treatment of GBM. The demonstration of improved OS in patients receiving neoadjuvant pembrolizumab is obviously substantial, however, the additional findings presented in this study also provide key information that may help in determining how and when the use of anti-PD-1 therapy can be most effective in the treatment of GBM.

An effective response to immunotherapy is largely dependent on three key components: (I) the immune system, 
(II) the tumor characteristics and (III) the unique interaction between the two. The successful use of checkpoint inhibitors, such as pembrolizumab, therefore, relies heavily on identifying characteristics of each of these components that predict which patients will be more likely to respond to certain therapies. The data presented by Cloughesy et al. suggests that patients in the neoadjuvant group, overall, experienced a better response to pembrolizumab, which is suggested to be a result of the upregulation of genes involved in the interferon induction pathway and $\mathrm{T}$ cell activity with concurrent suppression of genes involved in the cell cycle. However, close evaluation of Figure 2A,B suggests that there appear to be an overlap between the groups and that there are subsets of patients within each group that experience varied degrees of biological and clinical response (6). For example, there are several patients in the neoadjuvant group with low enrichment score in the interferon induction pathway as well as T-cell activation category and some others with increased enrichment score in the cell cycle/proliferation category, suggesting that altered tumor gene expression profile is not the only factor contributing to the effect seen in the neoadjuvant group. In addition, as evident from Figure 3, the level of PD-L1 expression and $\mathrm{CD}^{+} \mathrm{T}$-cell infiltration also varies within the group. While one cannot make any formal statements based on the interpretation of this graphic alone, the data presented herein clearly contains valuable information regarding the factors necessary for a clinically significant response to pembrolizumab. However, variability within the groups still exists and further evaluation of these subsets could prove to be crucial in guiding the determination of biological characteristics that warrant future investigation in a larger clinical trial.

In addition to understanding the inherent characteristics of individual patients that influence the response to immunotherapy, understanding the biological effects of immunotherapy is also critical. Cloughesy et al. addressed this important concept and their results demonstrated that, overall, neoadjuvant pembrolizumab results in the systemic expansion of tumor-specific T lymphocytes, an interferon$\gamma$-mediated immune response and the associated suppression of genes involved in the cell cycle and proliferation. Additionally, Cloughesy et al. demonstrated a significant increase in CTLA-4 on $\mathrm{CD}^{+}{ }^{+} \mathrm{T}$ cell clusters and focal increase in PD-L1 expression in response to neoadjuvant therapy, thereby supporting the investigation of combining neoadjuvant anti-PD-1 with other immunotherapies.

Despite the variability in the results reported by
Cloughesy et al. this study has opened a very important door in the world of GBM immunotherapy. Cloughesy et al. has not only demonstrated that pembrolizumab has a biological effect in the setting of recurrent GBM, but also that this biological effect is translating to clinical benefit. While the results of this study are promising, there still remains great variability in the response to neoadjuvant pembrolizumab and the efficacy of this treatment will be greatly dependent on the identification of subsets of patients who share similar characteristics capable of predicting the likelihood of response to anti-PD-1 therapy. Additionally, the use of anti-PD-1 therapy in GBM is unlikely to be as a single agent for any patient population and while Cloughesy et al. have demonstrated that neoadjuvant pembrolizumab triggers an anti-tumor immune response, it also results in the upregulation of other inhibitory checkpoint molecules, such as CTLA-4, CD276 and LAG3. There appears to be a specific subset of patients $(n=7)$ in the neoadjuvant group who demonstrated upregulation of both CTLA4 and LAG3 based on the data presented in Figure 2A,B, however, there is also great variability in the expression of these checkpoint molecules in both the neoadjuvant and adjuvant groups (6). Therefore, identifying specific subsets of patients will be critical for guiding the choice of effective combinations of immunotherapeutic agents.

Heterogeneity remains the greatest challenge in the successful treatment of GBM and with the emergence of immunotherapy, the hope was to eliminate this variable by exploiting the patients' immune system. Despite all of its promise, immunotherapy has yet to prove its efficacy against GBM. This study has restored the hope that immunotherapy has the potential to change the treatment of GBM and the challenge is now to identify the correct combination of therapies for the patients predicted to experience the most clinical benefit. As evident from this work, immune repertoire varies between patients and so does the immune response to the tumor leading to varying response to immunotherapy, thus immunotherapy might emerge as the final frontier of personalized medicine.

\section{Acknowledgments}

Funding: This work was supported by the NIH K08NS092895 grant (to M Dey).

\section{Footnote}

Provenance and Peer Review: This article is commissioned 
and reviewed by the Section Editor Xian-Xin Qiu, MD, PhD candidate [Shanghai Proton and Heavy Ion Center (SPHIC), a.k.a. the Proton and Heavy Ion Center of Fudan University Shanghai Cancer Center (FUSCC), Shanghai, China].

Conflicts of Interest: Both authors have completed the ICMJE uniform disclosure form (available at http://dx.doi. org/10.21037/tcr.2019.05.24). The authors have no conflicts of interest to declare.

Ethical Statement: The authors are accountable for all aspects of the work in ensuring that questions related to the accuracy or integrity of any part of the work are appropriately investigated and resolved.

Open Access Statement: This is an Open Access article distributed in accordance with the Creative Commons Attribution-NonCommercial-NoDerivs 4.0 International License (CC BY-NC-ND 4.0), which permits the noncommercial replication and distribution of the article with the strict proviso that no changes or edits are made and the original work is properly cited (including links to both the formal publication through the relevant DOI and the license). See: https://creativecommons.org/licenses/by-nc-nd/4.0/.

Cite this article as: Mitchell D, Dey M. Neoadjuvant antiPD-1 immunotherapy for recurrent glioblastoma. Transl Cancer Res 2019;8(Suppl 6):S577-S579. doi: 10.21037/ tcr.2019.05.24

\section{References}

1. Filley AC, Henriquez M, Dey M. Recurrent glioma clinical trial, CheckMate-143: the game is not over yet. Oncotarget 2017;8:91779-94.

2. Sun C, Mezzadra R, Schumacher TN. Regulation and Function of the PD-L1 Checkpoint. Immunity 2018;48:434-52.

3. Rini BI, Plimack ER, Stus V, et al. Pembrolizumab plus Axitinib versus Sunitinib for Advanced Renal-Cell Carcinoma. N Engl J Med 2019;380:1116-27.

4. Mok TSK, Wu YL, Kudaba I, et al. Pembrolizumab versus chemotherapy for previously untreated, PD-L1expressing, locally advanced or metastatic non-small-cell lung cancer (KEYNOTE-042): a randomised, open-label, controlled, phase 3 trial. Lancet 2019;393:1819-30.

5. Schachter J, Ribas A, Long GV, et al. Pembrolizumab versus ipilimumab for advanced melanoma: final overall survival results of a multicentre, randomised, open-label phase 3 study (KEYNOTE-006). Lancet 2017;390:1853-62.

6. Cloughesy TF, Mochizuki AY, Orpilla JR, et al. Neoadjuvant anti-PD-1 immunotherapy promotes a survival benefit with intratumoral and systemic immune responses in recurrent glioblastoma. Nat Med 2019;25:477-86. 